\title{
Lifetime of an adsorbate excitation modified by a tunable two-dimensional substrate
}

\author{
M. Wiesenmayer, ${ }^{1}$ M. Bauer, ${ }^{1, *}$ S. Mathias, ${ }^{2}$ M. Wessendorf, ${ }^{2}$ E. V. Chulkov, ${ }^{3,4}$ V. M. Silkin, ${ }^{3,4}$ A. G. Borisov, ${ }^{5,6}$ \\ J.-P. Gauyacq, ${ }^{5,6}$ P. M. Echenique, ${ }^{3,4}$ and M. Aeschlimann ${ }^{2}$ \\ ${ }^{1}$ Institut für Experimentelle und Angewandte Physik, Christian-Albrechts Universität zu Kiel, 24089 Kiel, Germany \\ ${ }^{2}$ Department of Physics and Research Center OPTIMAS, University of Kaiserslautern, 67663 Kaiserslautern, Germany \\ ${ }^{3}$ Departamento de Física de Materiales, Facultad de Ciencias Químicas, UPV/EHU and Centro Mixto CSIC-UPV/EHU, \\ Apartado1072, San Sebastián/Donostia, 20080 Basque Country, Spain \\ ${ }^{4}$ Donostia International Physics Center (DIPC), Paseo de Manuel Lardizibal, \\ 4 San Sebastián/Donostia, 20018 Basque Country, Spain \\ ${ }^{5}$ Laboratoire des Collisions Atomiques et Moléculaires, CNRS, Laboratorie des Collisions Atomiques et Moléculaires, UMR8525, \\ Bâtiment 351, 91405 Orsay Cedex, France \\ ${ }^{6}$ Laboratoire des Collisions Atomiques et Moléculaires, Université Paris-Sud, Laboratorie des Collisions Atomiques et Moléculaires, \\ UMR8525, Bâtiment 351, 91405 Orsay Cedex, France
}

(Received 30 September 2008; published 9 December 2008)

\begin{abstract}
The coupling efficiency between an adsorbate and a two-dimensional substrate is probed by real-time monitoring of the ultrafast charge transfer between a Cs atom and an ultrathin silver film of varying thickness adsorbed on a $\mathrm{Cu}(111)$ surface. For the first two monolayers of the silver film, a reduction in the resonance lifetime of the cesium $6 s-6 p$ hybrid state of approximately $35 \%$ is observed. When the silver coverage further increases, the resonance lifetime stays constant at a value close to the value for Cs adsorption on a bulk $\mathrm{Ag}(111)$ surface. Both the one-electron resonant and the multielectron inelastic contributions to the adsorbatesubstrate charge transfer are theoretically evaluated based on wave-packet propagation and $G W$ approximation. The results support the experimental findings and allow us to assign the observed dependence of the lifetime change in the multielectron inelastic contribution to the electron transfer rate between the Cs resonance and the very top atomic layers of the substrate.
\end{abstract}

DOI: 10.1103/PhysRevB.78.245410

PACS number(s): 68.43. $-\mathrm{h}, 68.65 . \mathrm{Fg}$, 82.53. $-\mathrm{k}$

\section{INTRODUCTION}

The chemical activity of a surface can become significantly altered when the thickness of the substrate approaches the nanometer length scale. One of the most striking examples is gold, which behaves almost inert if provided as a bulk and becomes highly reactive as nanodispersed aggregate on a substrate. ${ }^{1}$ In the past, experimental and theoretical model studies addressing the microscopic mechanisms behind such behavior of a surface have been undertaken on well-defined epitaxial ultrathin metal films. Generally, one finds that in the thickness regime below about 4 atomic layers modifications in the chemical activity are mainly due to the interaction of the film with the underlying substrate. Of relevance in this context there can be a structural mismatch between film and substrate resulting in a structural distortion and a consequent electronic perturbation at the surface. ${ }^{2-4}$ Another important mechanism influencing the charge transfer across substrate-film interfaces and thereby the chemical activity of the surface is the detailed electronic structure of the substrate. ${ }^{5,6}$ As the film thickness increases, the interaction with the substrate becomes less relevant for an adsorbate. Instead, the surface properties and chemical activity are strongly determined by the quantized energy spectrum of electron states [quantum-well states (QWSs)] due to electron confinement and interference in the ultrathin metal films. ${ }^{7}$ This quantization exhibits a clear thickness dependence enabling, for instance, a tailoring of the adsorbate-surface interaction. $^{8-13}$

This paper addresses the change in the lifetime of an adsorbate resonant electronic state (adsorbate resonance) as the thickness of a supporting ultrathin metal film is increased. Adsorbate resonances have attracted considerable attention due to their relevance in the context of electron-induced dynamical processes at surfaces such as resonant electronmolecule interactions in surface photochemistry, ${ }^{14-17}$ atom and molecular beam scattering, ${ }^{18-21}$ and dissociative molecular adsorption. ${ }^{22,23}$ The ultrafast decay dynamics of resonant electronic states of atoms and molecules in interaction with low-dimensional substrates have only been considered in theoretical studies so far. ${ }^{8,24}$

In our experimental and theoretical case studies, we investigate the lifetime of a resonance of an individual cesium atom adsorbed on epitaxial silver films up to 12 monolayer (ML) thickness, supported by a (111) oriented copper substrate. A Cs adsorbate on the (111) surface of a noble metal is an appealing system to study due to very long adsorbate resonance lifetimes that have been evidenced experimentally ${ }^{25,26}$ and theoretically ${ }^{27,28}$ in these systems. ${ }^{29}$ At low coverage, $\mathrm{Cs}$ adsorbs on a metal as a positive ion ${ }^{30,31}$ (see also a discussion in Ref. 29 and references therein). The long-lived $\mathrm{Cs}^{*}$ resonance state is associated with the transient capture of an electron by the $\mathrm{Cs}^{+}$adsorbate. The $\mathrm{Cs}^{*}$ resonance is a hybrid of the Cs $6 s$ and $6 p$ atomic orbitals, mainly in the $m=0$ symmetry subspace ${ }^{27-29,32}$ ( $m$ is the projection of the electron angular momentum along the atomic axis perpendicular to the surface). Due to the presence of a surfaceprojected band gap on the (111) surfaces of noble metals, the charge transfer between the adsorbate and the substrate is severely hampered, leading to long lifetimes of up to tens of femtoseconds. ${ }^{25-29}$ 
For the $\mathrm{Cs}$ adsorption on ultrathin $\mathrm{Ag}$ films on $\mathrm{Cu}(111)$, we observe experimentally a monotonic decrease in the $\mathrm{Cs}^{*}$ resonance lifetime within the first $3 \mathrm{ML}$ of silver from a value of $16 \pm 1.5$ fs for the pure copper substrate to a value of $10.5 \pm 1.5$ fs. For higher silver coverage no further change in the lifetime is observed. The results are well supported by theoretical calculations based on a joint wavepacket propagation and $G W$ approximation. This approach allows one to determine both the one-electron and multielectron contributions to the decay of the $\mathrm{Cs}^{*}$ resonance. The latter are found to dominate and are thus responsible for the variation in the $\mathrm{Cs}^{*}$ resonance lifetime with the $\mathrm{Ag}$ film thickness. In the context of surface chemical reactions we expect that such a tunability of charge-transfer rates in ultrathin films may be particularly useful in tailoring electroninduced processes.

\section{EXPERIMENTAL DETAILS}

\section{A. Experimental setup}

The angle-resolved photoemission and two-photon photoemission studies were conducted using a $150 \mathrm{~cm}$ hemispherical energy analyzer (SPECS Phoibos 150). The total energy resolution of the analyzer at the pass energy of $20 \mathrm{eV}$ used in these experiments is less than $20 \mathrm{meV}$, with an angular resolution of less than $0.15^{\circ}$. The electrons were collected with a two-dimensional (2D)-detection unit consisting of a microchannel plate, a phosphorus screen, and a charge coupled device (CCD) camera. This two-dimensional detector is able to record the electron emission spectra between $-7^{\circ}$ and $+7^{\circ}$ without rotating the sample. The laser system used for the two-photon photoemission experiments (2PPE) and the timeresolved two-photon photoemission experiments (TR-2PPE) is a mode-locked Ti:sapphire laser pumped by $6.9 \mathrm{~W}$ from a diode-pumped all-solid-state laser system. The system delivers linear-polarized transform-limited and squared hyperbolic secant temporally shaped pulses $(800 \mathrm{~nm}, 82 \mathrm{MHz}$ repetition rate, and 23 fs pulse width) with an energy of 15 $\mathrm{nJ} /$ pulse. The output of the Ti:sapphire laser is frequency doubled in a $0.2-\mathrm{mm}$-thick beta barium borate (BBO) crystal to produce pulses of $h \nu=3.1 \mathrm{eV}$ with a pulse width of $30 \mathrm{fs}$ [determined from autocorrelation measurements in 2PPE from the Shockley surface state (SS) of the $\mathrm{Cu}(111)$ surface]. A typical work function $\phi$ of the investigated surfaces between 3.5 and $4.5 \mathrm{eV}$ allows for photoemission within a twostep excitation process permitting the direct spectroscopic access to intermediate excited states between Fermi edge and vacuum level. In a conventional stroboscopic pump-probe configuration, it also enables us to probe the decay dynamics of the excited-state population at a temporal resolution of a few femtoseconds.

Reference spectra probing the occupied electronic structure of the surfaces were recorded in conventional onephoton photoemission (1PPE) using the $p$-polarized fourth harmonic light $(5.9 \mathrm{eV})$ of a second narrow bandwidth Ti:Sapphire oscillator generated by sequential frequency doubling in two BBO crystals (thickness: $0.2 \mathrm{~mm}$ each). For photoemission spectroscopy the UHV chamber is addition- ally equipped with a gas-discharge VUV-lamp operated at a photon energy of the HeI line $22 \mathrm{eV}$.

\section{B. Sample preparation and characterization}

\section{Ag overlayers on $\mathrm{Cu}(111)$}

The $\mathrm{Cu}(111)$ substrate used in this study was cleaned by repetitive sputtering $(10 \mathrm{~min}, 500 \mathrm{eV})$ and annealing cycles (15 min, 800K). The surface quality was checked by lowenergy electron diffraction (LEED), Auger spectroscopy, and the photoemission characteristics (energy and spectral width) of the Shockley surface state of the $\mathrm{Cu}(111)$ surface. Silver was evaporated from a Knudsen cell-type evaporation source at a rate of about $1 \mathrm{ML} / \mathrm{min}$. During evaporation, the sample temperature was held at $300 \mathrm{~K}$. At room temperature silver grows in a Stranski-Krastanov mode on $\mathrm{Cu}(111)$ : a layer by layer growth is observed for the first two ML, and at higher coverages three-dimensional (3D) island growth appears. ${ }^{33-36}$ The appearance of a $9 \times 9$ superstructure of the deposited silver films for low coverage has been reported in a number of publications ${ }^{32,37,38}$ and was also clearly observable by LEED in the present study. The development of the superstructure arises to compensate the mismatch of about $13 \%$ in the lattice constants between a (111)-oriented silver overlayer and the $\mathrm{Cu}(111)$ substrate. The strain is relieved by a lattice of dislocation loops in the $\mathrm{Cu}(111)$ substrate. ${ }^{39}$ Starting at a thickness of about $14 \mathrm{ML}$, quantum-well states evolve in the $\mathrm{Ag} / \mathrm{Cu}(111)$ system. $^{40-43}$ The appearance of these states is possible due to the energetic mismatch of the $s p$ band gaps between copper and silver close to the $\Gamma-L$ direction. $^{7}$

The film quality for silver film thicknesses $\Theta>2$ ML has been improved considerably by a short annealing treatment at $T=600 \mathrm{~K}$. This procedure transforms the 3D island structure into a homogeneous flat film, characterized by narrow almost purely Lorenzian-shaped photoemission peaks from the Shockley surface state and from the quantum-well states. ${ }^{40,43}$ A drawback of this preparation step is a characteristic bifurcation of the film into areas covered by a (homogeneous) thick silver layer and areas covered by only $2 \mathrm{ML}$ of silver. ${ }^{43}$ However, as we will see later, the bifurcation mode of the film barely affects the interpretation of our timeresolved experimental results.

The binding energy of the Shockley surface state for the $\mathrm{Ag} / \mathrm{Cu}(111)$ surface depends critically on the silver film thickness, where the highest sensitivity is observed in the coverage regime between 0 and 5 ML. ${ }^{36,38,43,44}$ This energy shift results from the finite penetration depth (a few monolayer) of the surface state into the bulk and the consequent interaction with the $\mathrm{Ag} / \mathrm{Cu}(111)$ interface and the $\mathrm{Cu}(111)$ substrate in this thickness regime. Figure 1(a) shows binding-energy values of the surface state as function of silver film thickness at $300 \mathrm{~K}$ as obtained in an earlier photoemission experiment. ${ }^{43}$ In that work we paid particular attention to correct for systematic errors in the determination of the surface state energy arising from the 3D growth of the silver film. The binding-energy values of the surface state as well as the thickness-dependent work function values determined from the low-energy onset of the photoemission spec- 

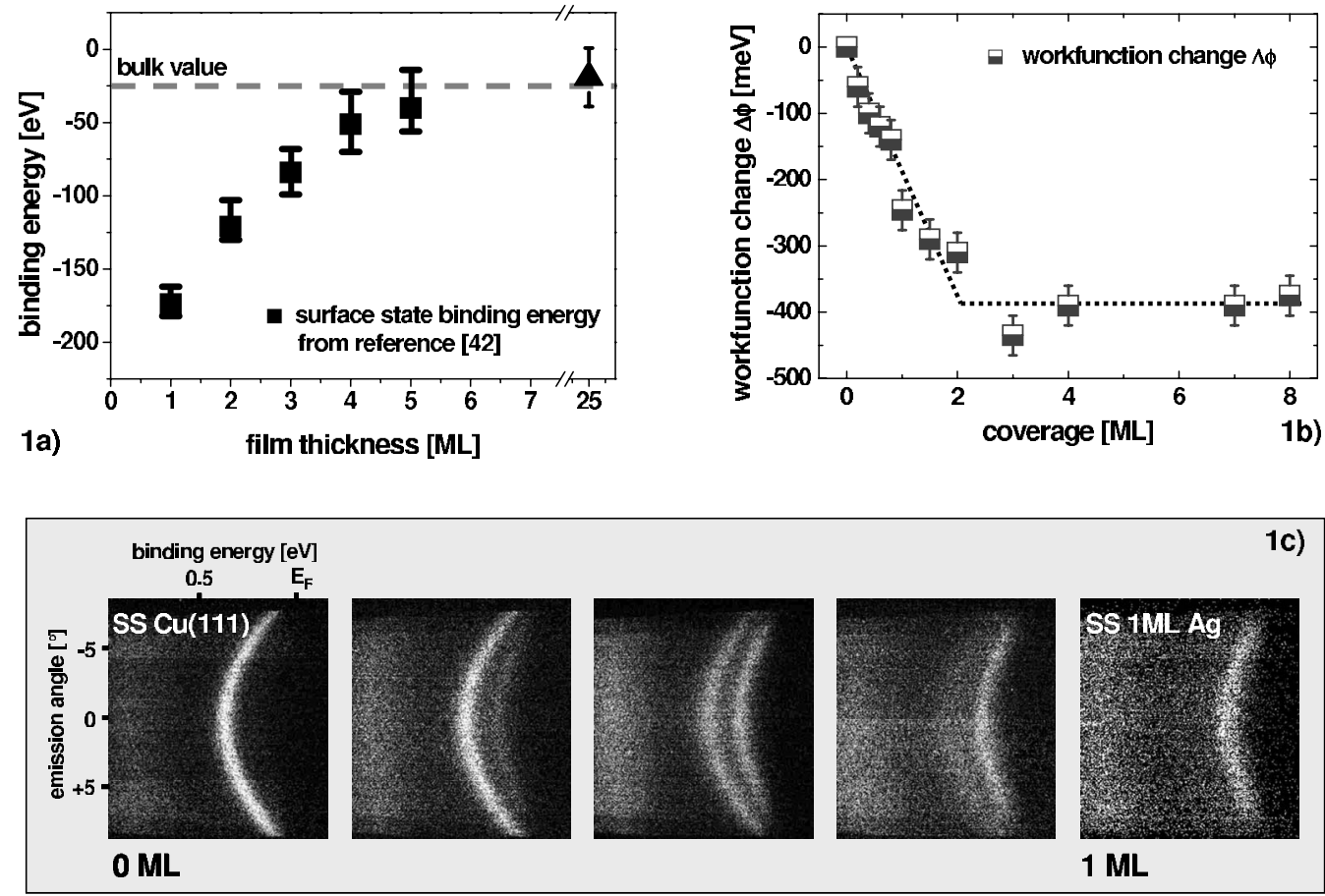

FIG. 1. (a) Surface state binding energy of $\mathrm{Ag} / \mathrm{Cu}(111)$ as function of silver film thickness (data taken from Ref. 43). (b) Change in the work function as function of silver film thickness determined from the onset of the photoemission spectra recorded for different coverages. (c) 1PPE $E\left(k_{\|}\right)$intensity maps recorded with the HeI line of the VUV-lamp for increasing silver coverage in the thickness regime between 0 and $1 \mathrm{ML}$. As the thickness increases, the Shockley surface state of the 1-ML-thick silver areas appear in the spectrum. For a closed $1 \mathrm{ML}$ film (very right intensity map), the signal of the $\mathrm{Cu}(111)$ Shockley surface state has disappeared.

tra [Fig. 1(b)] are used as parameters for the theoretical modeling of the $\mathrm{Ag} / \mathrm{Cu}(111)$ system to correctly mimic the modifications in the surface potential arising from the successive deposition of the silver layers.

The thickness calibration required for the thin-film preparation was deduced from a measurement of the intensity ratio between the Shockley surface state of the pure $\mathrm{Cu}(111)$ substrate and the Shockley surface state of a single layer of silver in $1 \mathrm{PPE}$ as the silver coverage increases from 0 to 1 monolayer. ${ }^{35,43}$ Figure $1(\mathrm{c})$ shows corresponding 1PPE $E\left(k_{\|}\right)$ intensity maps. Due to the growth mode of the silver film, a completed first monolayer corresponds to the coverage where the emission from the $\mathrm{Cu}$ surface state has just disappeared. For the thickness regime $\Theta>5 \mathrm{ML}$, additional experiments were performed on a silver wedge deposited on the $\mathrm{Cu}(111)$ substrate. For this kind of sample a thickness calibration was achieved utilizing the thickness-dependent binding-energy values of the silver film quantum-well states as well as of the Shockley surface state [Fig. 1(a)]. These characteristics allow for an accurate determination of the film thickness at the wedge for $\Theta>14$ ML regions (by means of the QWS) and for $\Theta<7 \mathrm{ML}$ regions (by means of the Shockley surface state). By a linear interpolation from these two regions to the intermediate positions in the wedge, we finally localized the sample positions corresponding to the film thickness values between 8 and 13 ML.

\section{Cesium adsorption}

The adsorption of alkali atoms on metal surfaces has been investigated thoroughly in the past from a theoretical as well as from an experimental point of view. ${ }^{45}$ In the context of the dynamics of charge-transfer processes, cesium adsorption on noble-metal surfaces has attracted considerable attention due to the formation of an exceptionally long-living resonance, first reported for the $\mathrm{Cs} / \mathrm{Cu}(111)$ system. ${ }^{25-29}$ As discussed earlier, the $\mathrm{Cs}^{*}$ resonance corresponds to the transient capture of an electron in a $6 s-6 p$ hybrid by a positively charged $\mathrm{Cs}^{+}$adsorbate. In the limit of the adsorption of an individual cesium atom on a noble metal, the resonance energy $E_{\text {Res }}$ is about $3 \mathrm{eV}$ above the Fermi energy, almost independent of noble-metal species and surface orientation. ${ }^{28,46,47}$ With increasing Cs coverage, the resonance energy shifts toward the Fermi energy, a behavior arising from the interaction between the excited $\mathrm{Cs}^{*}$ state and the dipole layer formed by the surrounding alkali adsorbates. ${ }^{27}$

Figure 2 shows 2 PPE $E\left(k_{\|}\right)$intensity maps for an increasing coverage of $\mathrm{Cs}$ atoms adsorbed on a silver film. The $\mathrm{Cs}$ was deposited from a resistive heated getter source. In these examples, the Cs coverage ranges between a value of 0.03 to $0.05 \mathrm{ML}$. The downshift of the $\mathrm{Cs}^{*}$ resonance as the coverage increases is clearly visible. Furthermore, no dispersion of the $\mathrm{Cs}^{*}$ resonance is noticeable, indicating a negligible wavefunction overlap between neighboring $\mathrm{Cs}$ atoms at these coverages.

Figure 3(a) shows a 2PPE energy distribution curve (EDC) and a 1PPE EDC at the $\bar{\Gamma}$ point for an Ag film on $\mathrm{Cu}(111)$ covered with approximately $0.05 \mathrm{ML}$ of $\mathrm{Cs}$ as deduced from an $E\left(k_{\|}\right)$intensity map. For comparison, the top viewgraph shows the corresponding 1PPE EDC of the surface without the alkali adsorbate. The data were recorded at 


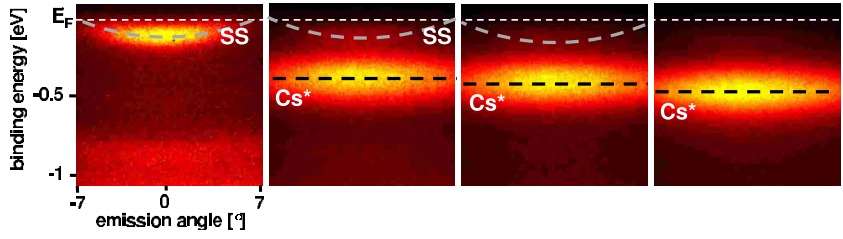

FIG. 2. (Color online) 2PPE $E\left(k_{\|}\right)$intensity maps for Cs adsorption on a silver film grown on $\mathrm{Cu}(111)$ at increasing coverage. (a) 2PPE map without Cs showing the dispersive Shockley SS of the silver overlayer. (b) Even for very small Cs coverages a clear nondispersive signature from the Cs resonance state (Cs Res) appears in the spectrum; still visible is some residual from the Shockley surface state. [(c) and (d)] As the Cs coverage increases, the Cs resonance shifts down in energy.

room temperature following a $600 \mathrm{~K}$ flash of the silverwedge sample. Figure 3(b) illustrates the energetics of the $1 \mathrm{PPE}$ and 2PPE excitation schemes. Both 1PPE spectra are dominated by two Shockley surface state peaks (SS1 and SS2) characteristic for the bifurcation mode of the annealed silver film. For the alkali-free surface the binding energy of the SS1 peak is $125 \mathrm{meV}$ and is a characteristic for a 2-MLthick film of silver. The binding energy of $25 \mathrm{meV}$ of SS2 agrees well with the value reported for the Shockley surface state of a bulk $\mathrm{Ag}(111)$ sample. ${ }^{48}$ The interpolation procedure
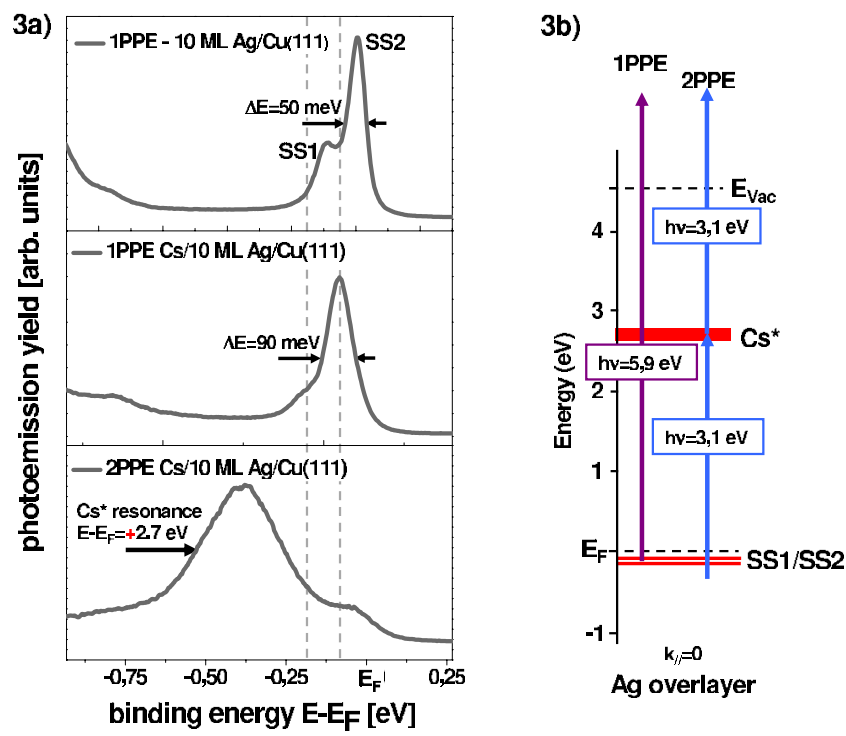

FIG. 3. (Color online) (a) 1PPE and 2PPE excitation schemes illustrating the appearance of the SS peaks and the Cs peak in the corresponding spectra. Note that the population of the $\mathrm{Cs}^{*}$ resonance state in the $2 \mathrm{PPE}$ process does not arise from a resonant excitation from the surface states SS1 and SS2. (b) 1PPE and 2PPE spectra of an uncesiated (top) and a cesiated surface (middle and bottom spectra, coverage $\sim 0.05 \mathrm{ML}$ ) of a 10-ML-thick silver layer grown on $\mathrm{Cu}(111)$ at room temperature after flashing to $600 \mathrm{~K}$. The SS1 feature is the Shockley surface state of the surface areas covered with 2ML of silver, and the SS2 feature is the Shockley surface state of areas covered with $10 \mathrm{ML}$ of silver. The energy scale refers to the binding energy with respect to the occupied density of states. Note that the Cs resonance state is an excited state located at $E-E_{F}=+2.7 \mathrm{eV}$. from the border regions of the wedge, described above, enables us to determine the actual silver coverage of the thick film areas as $10 \mathrm{ML}$. The 1PPE EDC of the Cs-covered surface shows that the alkali adsorption affects both surface state peaks considerably: an energy shift of the states of about $50 \mathrm{meV}$ toward higher binding energies reflects the electron donation from the Cs adsorbate to the surface state. The obvious broadening of both states ( $\triangle \mathrm{FWHM}$ $\approx 40 \mathrm{meV}$, where FWHM stands for full width at half maximum) arises from the scattering of the freely propagating surface state electrons with the adsorbed Cs atoms as previously observed for other adsorbate systems. ${ }^{49-53}$ As both surface states are similarly quantitatively modified, we conclude that the cesium coverage at the 2 and $10 \mathrm{ML}$ areas is about equal.

In the 2PPE EDC [Fig. 3(a), bottom viewgraph] the two surface state peaks are not present. The strong quenching of the surface state emission in 2PPE seems to be a silver surface specific effect and has been observed by other groups ${ }^{54,55}$ Clearly visible in the spectrum is however the Cs $6 s-6 p$ resonance state at an excitation energy $E-E_{F}$ $=2.73 \pm 0.03 \mathrm{eV}$ [note that the energy scale given in Fig. 3 (a) refers to the binding energy of the occupied states]. For the time-resolved experiments the Cs coverage (and in consequence the $\mathrm{Cs}^{*}$ resonance energy) was tuned in such a way that a resonant excitation from the two surface states is avoided. The energetic mismatch between Shockley surface states (acting as the initial state of a 2PPE process) and the $\mathrm{Cs}^{*}$ resonance (as intermediate state) is evident from the comparison of the 1PPE EDC [Fig. 3(a), middle] and the 2PPE EDC [Fig. 3(a); bottom] of the cesiated sample under consideration of the excitation schemes illustrated in Fig. 3 (b). The measured 2PPE signal (as well as the TR 2PPE signal discussed below) from the $\mathrm{Cs}^{*}$ resonance signal is therefore an average of the signal from the 2- and 10-MLthick films. The relative contributions from each layer can be estimated by the intensity ratio of the two corresponding surface states. For the example shown in Fig. 3(a) we find a ratio $I_{10 \mathrm{ML}} / I_{2} \mathrm{ML}=3.3 / 1$. Nevertheless, as we will later see, this signal mixing will not affect the relevant conclusions made from our experiment.

\section{THEORY}

The aim of the theoretical study is to compute the energy $E_{\text {Res }}$ and the decay rate $\Gamma$ of the $\mathrm{Cs}^{*}$ resonance state on a thin $\mathrm{Ag}$ film of variable thickness. Two different decay routes exist for the $\mathrm{Cs}^{*}$ resonance: one-electron energy-conserving transitions (resonant decay), in which the $\mathrm{Cs}^{*}$ outer electron is transferred into bulk states of the same energy, and inelastic multielectron transitions, in which the inelastic interaction between the $\mathrm{Cs}^{*}$ electron and a substrate electron leads to two excited electrons in the substrate. The two contributions to the decay rate are computed separately using an approach described in Refs. 27-29 and 56. Only the general idea of the method is presented here, together with the points specific to the present study.

Basically, the approach consists of defining the potential, in which the outer electron of the $\mathrm{Cs}^{*}$ resonance is evolving. 
The corresponding one-electron Hamiltonian for a single adsorbate on the surface is written as

$$
H=T+V_{e \text {-Ads }}+\Delta V_{e-\text { Surf }}+V_{e \text {-Surf }},
$$

where $T$ is the electron kinetic energy.

$V_{e-\text { Ads }}$ is the electron interaction with the adsorbate core. As mentioned above, at low Cs coverage, Cs adsorbs on a metal as a positive ion $^{30,31}$ (see also a discussion and references in Ref. 29) and the long-lived $\mathrm{Cs}^{*}$ resonance state corresponds to the transient capture of an electron by the $\mathrm{Cs}^{+}$ adsorbate. The long-lived $\mathrm{Cs}^{*}$ resonance is a hybrid of $6 s$ and $6 p$ atomic states. $V_{e \text {-Ads }}$ is then represented by the electronfree $\mathrm{Cs}^{+}$interaction as determined in atomic physics studies, ${ }^{57}$ it contains local and nonlocal terms in KleinmanBylander form ${ }^{58}$ (see Ref. 53 for an explicit definition). The adsorption distance of $\mathrm{Cs}$ on $\mathrm{Cu}(111)$ was taken equal to $3.5 a_{0}$, measured from the metal image reference plane ${ }^{28}$ and to $3.75 a_{0}$ for $\mathrm{Ag}(111){ }^{59}$ For the $\mathrm{Ag}$ thin layers, the adsorption distance was taken equal to that on $\operatorname{Ag}(111)$ (changes in the adsorption distance by a fraction of $a_{0}$ is not expected to bring a large variation in the $\mathrm{Cs}^{*}$ resonance decay rate; the adsorption energy is expected to vary slowly, approximately following an image charge-surface interaction law).

$\Delta V_{e \text {-Surf }}$ is the modification of the electron-surface interaction potential induced by the presence of the charged adsorbate. It was taken equal to the classical image charge interaction between the active electron and the image of the adsorbate ion core.

$V_{e-\text { Surf }}$ is the electron-surface interaction potential; it is chosen to be only a function of $z$, the electron-coordinate perpendicular to the surface. For pristine $\mathrm{Cu}(111)$ and $\mathrm{Ag}(111)$ surfaces this potential has been taken from Ref. 60 with parameters fitted to the measured low-temperature energy of the $\bar{\Gamma}$ surface state ${ }^{61}$ and the first image-potential state. ${ }^{60}$ At a coverage of $1 \mathrm{ML}$ of $\mathrm{Ag}$ on $\mathrm{Cu}(111)$ we use a potential proposed in Ref. 62 with parameters fitted to the surface state energy and the first image-potential state energy. ${ }^{35}$ For the thicker Ag films the potential has been modified by using the bulk Ag potential ${ }^{37}$ for internal $\mathrm{Ag}$ layers and the form in Ref. 62 for the outermost Ag layer with parameters which reproduce the experimental energy of the surface state and the first image-potential state ${ }^{35}$ for all Ag films of interest.

The time-dependent Schrödinger equation with the Hamiltonian (1) is solved by wave-packet propagation on a grid of points (see Refs. 56 and 63 for details). With the above choice of potentials, the system is invariant by rotation around the $z$-axis normal to the surface, which goes through the adsorbate center. The projection of the electron momentum on this axis, $m$, is a good quantum number. Since the $\mathrm{Cs}^{*}$ state is a $6 s-6 p$ hybrid of $m=0$ symmetry, the problem has been solved in cylindrical coordinates within the $m=0$ symmetry. From the time dependence of the wave packet, one can extract the energy of the resonance $E_{\text {Res }}$, its resonant decay rate $\Gamma_{1 e}$, and the corresponding wave function $\Phi_{\text {Res }}{ }^{63}$ The problem treated by the Hamiltonian (1) is monoelectronic so that the decay rate $\Gamma_{1 e}$ obtained in this calculation only concerns the one-electron decay by resonant transitions from the $\mathrm{Cs}^{*}$ resonance state into substrate states of the same

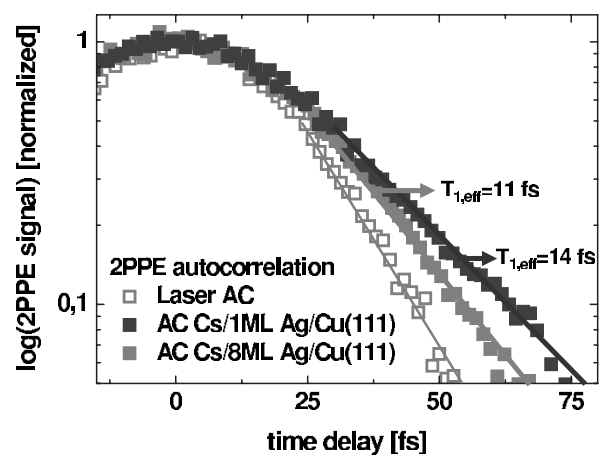

FIG. 4. Comparison of time-resolved 2PPE autocorrelation traces measured for the excitation of the $\mathrm{Cs}^{*}$ resonance at two different silver film thicknesses ( 1 and $8 \mathrm{ML}$ ). For reference also the laser autocorrelation determined by 2PPE from the $\mathrm{Cu}(111)$ Shockley surface state is shown. The difference in the two Cs traces arises from the variation in the resonance lifetime as the silver film thickness increases. $T_{1 \text {,eff }}$ are the effective population relaxation times deduced from the experimental autocorrelation traces (details see text).

energy. The $\mathrm{Cs}^{*}$ wave function $\Phi_{\mathrm{Res}}$, obtained in the oneelectron wave-packet calculation is then used to obtain the multielectron contribution to the $\mathrm{Cs}^{*}$ decay, $\Gamma_{e e}$, via a perturbative many-body approach, based on the $G W$ approximation. ${ }^{64}$ Details of the calculation of $\Gamma_{e e}$ are given in Refs. 28, 56, and 65.

The present theoretical study corresponds to a single Cs adsorbate on the surface and therefore should be relevant for discussing experimental results with a very low Cs coverage. Theoretical analysis ${ }^{27,29}$ showed that increasing the coverage leads to a small change in the $\Gamma_{1 e}$ rate and to a shift $\Delta E$ of the resonance energy toward Fermi level. $\Delta E$ was shown ${ }^{27}$ to vary proportionally to $(\Delta \phi)^{3 / 2}$, where $\Delta \phi$ is the surface work function change induced by the adsorbates.

\section{LIFETIME OF THE Cs* RESONANCE: EXPERIMENT AND THEORY}

Figure 4 shows experimental 2PPE autocorrelation traces recorded with $p$-polarized light for the excitation of the $\mathrm{Cs}^{*}$ resonance for adsorption on a 1-ML-thick and a 8-ML-thick silver films. For reference, the laser autocorrelation trace, determined by off-resonant 2PPE excitation from the Shockley surface state of the silver film without adsorbate, is also shown. Note that the data are displayed in a semilogarithmic plot and that the constant signal background arising from the 2PPE signal of two independent laser pulses has been subtracted. For these measurements, a series of 2PPE $E\left(k_{\|}\right)$intensity maps have been recorded for various time delays between two identical incident $400 \mathrm{~nm}$ light pulses. From this data set, phase-averaged autocorrelation traces are extracted afterwards for a selected $E\left(k_{\|}\right)$region of interest, i.e., in the present case at the $\mathrm{Cs}^{*}$ resonance energy over an extended $k_{\|}$ regime in the vicinity of the $\bar{\Gamma}$ point. The clear broadening of the two autocorrelation traces from the $\mathrm{Cs}^{*}$ resonance in comparison to the laser autocorrelation trace illustrates the significant contribution to the measured signal due to the 


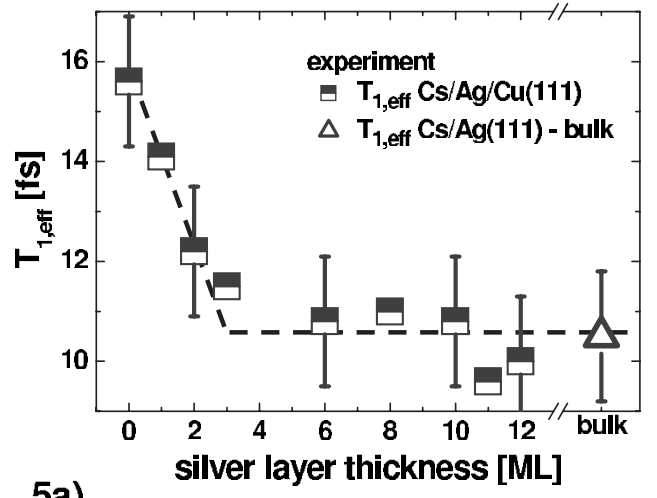

5a)

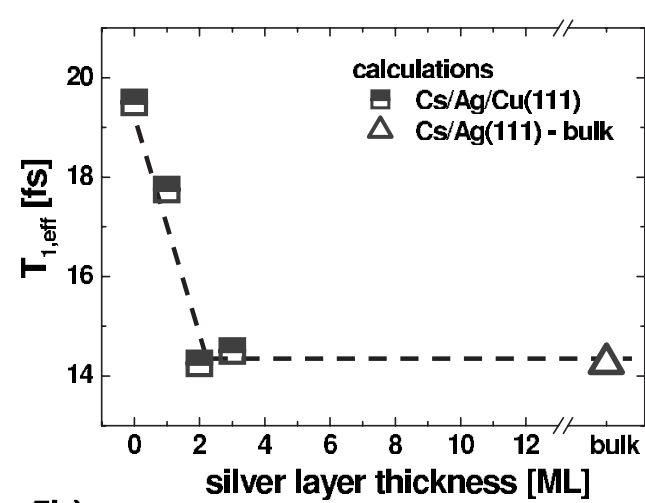

5b)

FIG. 5. Cs* resonance effective lifetime as function of silver film thickness: (a) $T_{1, \text { eff }}$ as measured in the TR-2PPE experiment and (b) $T_{1, \text { eff }}$ as determined from theory.

short but finite lifetime of the cesium resonance. Furthermore, the obvious difference between the two cesium autocorrelation traces is evidence of a variation in the $\mathrm{Cs}^{*}$ resonance lifetime as the silver film thickness changes.

An absolute quantification of the detailed dynamics associated with the $\mathrm{Cs}^{*}$ resonance from $2 \mathrm{PPE}$ correlation measurements is a complex task. The energy spectrum of the emitted electron and its time dependence is governed not only by the lifetime of the resonance, but also by the motion of the adsorbate away from the surface induced by the laser excitation. ${ }^{66-69}$ A quantitative data evaluation is further complicated by effects such as the thermal vibrational excitation of the adsorbate-substrate bond, the actual shape of the exciting laser pulse, and the coupling between electronic excitation and nuclear motion. ${ }^{66-69}$ Therefore extracting the resonance lifetime from the experimental time-resolved 2PPE signal, while taking the $\mathrm{Cs}$ motion into account is not straightforward, and we resort to the definition of an effective population relaxation time.

The quantification of the effective $\mathrm{Cs}^{*}$ population relaxation time is achieved by following an evaluation method proposed and exemplified in a recent publication ${ }^{69}$ and inspired by a discussion in Ref. 70. This approach allows one to extract a characteristic time $T_{1 \text {,eff }}$ as a measure for an effective survival time for the $\mathrm{Cs}^{*}$ resonance, modified by the cesium motion. Even though the actual value of $T_{1 \text {,eff }}$ depends on the experimental conditions (laser band width and energy resolution), the model enables a satisfactory comparison between experimental data and theoretical results by defining effective $T_{1 \text {,eff }}$ times exactly the same in both cases. As will be shown later, $T_{1 \text {,eff }}$ is under the present experimental conditions approximately 0.75 times the resonance lifetime $\tau$. We evaluate $T_{1, \text { eff }}$ from the 2PPE signal as a function of time delay as follows: a series of exponential decay functions is calculated for different time constants and convoluted with the laser autocorrelation trace. These traces are then quantitatively compared within a $\chi^{2}$ test to the autocorrelation traces of the $\mathrm{Cs}^{*}$ resonance. We restrict this comparison to the time delay range between 0 and $100 \mathrm{fs}$. The trace yielding the least $\chi^{2}$ value gives the best value for $T_{1, \text { eff }}$. The same procedure is applied to the theoretical 2PPE signal computed using the present potential-energy curves and lifetimes of the $\mathrm{Cs}^{*}$ resonance and the modeling from Ref. 69.
Experimental $T_{1, \text { eff }}$ values for Cs adsorption on a silver film thickness varying between 0 and $12 \mathrm{ML}$ as well as for bulk $\mathrm{Ag}(111)$ are displayed in Fig. 5(a). Within the first few monolayers of silver the lifetime of the $\mathrm{Cs}^{*}$ resonance decreases almost linearly with the Ag film thickness. Starting from $T_{1, \mathrm{eff}} \approx 16 \pm 1.5 \mathrm{fs}$ for $\mathrm{Cs}$ adsorption at the pure $\mathrm{Cu}(111)$ substrate the lifetime decreases to $\mathrm{T}_{1 \text {,eff }}$ $\approx 10.5 \pm 1.5 \mathrm{fs}$, a value reached at about $3-4 \mathrm{ML}$. For higher silver coverage, no further significant change in the resonance lifetime could be detected, at least for the investigated thickness range up to $12 \mathrm{ML}$. Even more, the $T_{1 \text {,eff }}$ value found in this thickness regime agrees almost perfectly with $T_{1 \text {,eff }}$ measured for the adsorption of $\mathrm{Cs}$ on a clean $\operatorname{Ag}(111)$ sample [open triangle in Fig. 5(a)]. We conclude that the relevant modifications in the substrate electronic structure responsible for the population decay of the Cs excitation takes place within the first three monolayers of silver. Already at silver coverage $\Theta \approx 4 \mathrm{ML}$ the film acts on the electron dynamics of the $\mathrm{Cs}^{*}$ resonance very similarly to a $\operatorname{Ag}(111)$ bulk substrate surface.

For a laser-pulse width of $30 \mathrm{fs}$, it may seem to be challenging to resolve a lifetime difference as small as 2-4 fs. However, note the clear difference in the 2PPE autocorrelation signal shown in Fig. 4 which clearly demonstrates our subpulse resolution. Furthermore, we would like to point out that the first three data points in Fig. 5(a) are an average from three independent experimental runs on separately prepared silver films, and the scatter of the respective lifetime determines the error bars. Also the lifetime values for coverages $\geq 3$ ML have been collected within two different experimental runs using a silver-wedge sample as well as individual films of corresponding thickness. The latter data points ( $\geq 3 \mathrm{ML}$ ) need some additional comments. As mentioned above, in this thickness regime, a short annealing was applied after evaporation to homogenize the film. As a drawback, this procedure resulted in a bifurcation of the film morphology giving rise to the presence of extended areas of 2 ML thickness only. The displayed lifetime values therefore represent an average for adsorption of Cs on a 2-ML-thick silver film and a silver film corresponding to the labeled thickness value. Note that the contribution from the $2 \mathrm{ML}$ film decreases with increasing film thickness. As we do not observe any change in $T_{1 \text {,eff }}$ over the entire thickness regime 
TABLE I. Theoretical results for the resonance energy $E_{\text {Res }}$, one-electron decay rate $\Gamma_{1 e}$, multielectron rate $\Gamma_{e e}$, partial contribution from the surface state $\Gamma_{e e}^{\mathrm{SS}}$, total decay rate $\Gamma=\Gamma_{1 e}+\Gamma_{e e}$, lifetime $\tau=1 / \Gamma$, and effective lifetime $T_{1, \text { eff }}$ for the $\mathrm{Cs}^{*}$ resonance for different substrates: clean $\mathrm{Cu}(111)$, clean $\operatorname{Ag}(111)$, and $\mathrm{Cu}(111)$ covered by 1,2 , or $3 \mathrm{ML}$ of $\mathrm{Ag}$. The Cs coverage of the surface is asymptotically small. The energies are given in $\mathrm{eV}$ with respect to vacuum level. The decay rates are given in meV and the lifetime in femtosecond. $\Gamma_{e e}^{\mathrm{SS}}$ is the contribution to $\Gamma_{e e}$ due to the interaction with the Shockley surface state.

\begin{tabular}{lccccccc}
\hline \hline & $\begin{array}{c}\text { Energy, } E_{\text {Res }} \\
(\mathrm{eV})\end{array}$ & $\begin{array}{c}\Gamma_{1 e} \\
(\mathrm{meV})\end{array}$ & $\begin{array}{c}\Gamma_{e e} \\
(\mathrm{meV})\end{array}$ & $\begin{array}{c}\Gamma_{e e}^{\mathrm{SS}} \\
(\mathrm{meV})\end{array}$ & $\begin{array}{c}\Gamma \\
(\mathrm{meV})\end{array}$ & $\begin{array}{c}\tau \\
(\mathrm{fs})\end{array}$ & $\begin{array}{c}T_{1, \text { eff }} \\
(\mathrm{fs})\end{array}$ \\
\hline $\mathrm{Clean} \mathrm{Cu}(111)^{\mathrm{a}}$ & -1.952 & 7 & 16.5 & 6.5 & 23.5 & 28 & 19.5 \\
$1 \mathrm{ML} \mathrm{Ag} / \mathrm{Cu}(111)$ & -1.956 & 4.7 & 21.2 & 12.5 & 25.9 & 25.4 & 17.8 \\
$2 \mathrm{ML} \mathrm{Ag} / \mathrm{Cu}(111)$ & -1.970 & 7.8 & 28.4 & 15.2 & 36.2 & 18.2 & 14.3 \\
$3 \mathrm{ML} \mathrm{Ag} / \mathrm{Cu}(111)$ & -1.975 & 8.5 & 26.2 & 12.0 & 34.7 & 19 & 14.5 \\
Clean Ag$(111)$ & -1.967 & 8.9 & 26.7 & 11.1 & 35.6 & 18.5 & 14.3 \\
\hline \hline
\end{tabular}

${ }^{\mathrm{a} R e f e r e n c e ~} 28$.

$>3 \mathrm{ML}$, we conclude that the 2 ML contribution to the signal is negligible, at least in comparison to the experimental error in our measurements. Only for the $3 \mathrm{ML}$ data point one may find some indication for such a (2 ML induced) systematic deviation toward a slightly higher lifetime value, i.e., for a rounding of the coverage dependence of $T_{1, \text { eff }}$.

The results of our corresponding calculations of the $\mathrm{Cs} /$ $\mathrm{Ag} / \mathrm{Cu}(111)$ system (energy $E_{\text {Res}}$, one-electron decay rate $\Gamma_{1 e}$, multielectron rate $\Gamma_{e e}$, partial contribution from the surface state $\Gamma_{e e}^{\mathrm{SS}}$, total decay rate $\Gamma=\Gamma_{1 e}+\Gamma_{e e}$, resonance lifetime $\tau=1 / \Gamma$, and effective lifetime $T_{1, \text { eff }}$ ) are summarized in Table I. The energy of the $\mathrm{Cs}^{*}$ resonance only slightly varies from one system to the other since the same $\mathrm{Cs}^{*}$ resonance is considered that does not vary much from one system to the other except for a small variation in the adsorption distance between $\mathrm{Ag}$ and $\mathrm{Cu}$. As for the resonance lifetime $\tau$, it is seen to significantly decrease from the $\mathrm{Cu}(111)$ substrate to the $\mathrm{Ag}(111)$ substrate; it varies monotonically when the $\mathrm{Ag}$ layer thickness is increased from 0 to $2 \mathrm{ML}$. The transition from $\mathrm{Cu}$ to the $\mathrm{Ag}$ bulk lifetime value is very sharp; it is basically finished at $2 \mathrm{ML}$; only the $1 \mathrm{ML}$ Ag layer exhibits an actual intermediate behavior. It also appears that, for all the systems studied, the multielectron decay rate dominates the decay of the $\mathrm{Cs}^{*}$ state so that the variation in lifetime along the $n \mathrm{ML} \mathrm{Ag/Cu(111)} \mathrm{system} \mathrm{series} \mathrm{has} \mathrm{to} \mathrm{be} \mathrm{attributed}$ to the difference of the multielectron decay rate in the case of $\mathrm{Ag}$ and $\mathrm{Cu}$.

One can stress that the dominance of the $\Gamma_{e e}$ term in the decay rate is linked with the long lifetime of the resonance. Usually, one would expect the one-electron term $\Gamma_{1 e}$ to dominate over the multielectron terms. However, in these systems, the $\mathrm{Cs}^{*}$ resonance is degenerate with the surface projected band gap of $\mathrm{Cu}(111)$ and $\mathrm{Ag}(111)$ so that the substrate states, which are normally the most active in the resonant charge transfer, are absent in all the systems investigated here. This effect associated with the polarization of the electronic cloud leads to a drastic reduction in the $\Gamma_{1 e}$ rate (typically 2 orders of magnitude on these substrates) and therefore to the dominance of multielectron effects and to a long lifetime. ${ }^{27-29}$

$T_{1 \text {,eff }}$ values extracted from the modeling of the $2 \mathrm{PPE}-$ signal (hyperbolic squared secant-shaped laser pulse with a width (FWHM) of $30 \mathrm{fs}$ ) are displayed for comparison with the experimental values of $T_{1 \text {,eff }}$ in Fig. 5(b) for a silver film thickness varying between 0 and 3 ML and for bulk $\mathrm{Ag}(111)$. It appears that the $T_{1, \text { eff }}$ varies approximately proportionally to the lifetime $\tau$ with a ratio of the order of 0.75 as already mentioned above (this ratio decreases slightly when the lifetime increases). This shortening of the effective time $T_{1 \text {,eff }}$ compared to the real population lifetime $\tau$ is similar to that found in Ref. 69 (with different laser shapes ${ }^{71}$ ). It is a direct consequence of the Cs motion induced by the electronic excitation: when the adsorbate moves away, the $\mathrm{Cs}^{*}$ resonance energy decreases and the corresponding energy of the photoemitted electron also decreases and moves out of the experimental detection window.

From a qualitative point of view, the theoretical calculations (both $\tau$ and $T_{1 \text {,eff }}$ ) reproduce the experimental behavior strikingly well. Again we observe a quasilinear decrease in the lifetime within the first few ML. The sharpness of the transition is slightly stronger in the theoretical results than experimentally observed. Typically the critical transition coverage is at $2 \mathrm{ML}$ in the theoretical results and around 2-3 ML in the experimental data. The comparison of the 2 and 3 ML theoretical value with the $\mathrm{Ag}(111)$ bulk value strongly suggests that no further change in the lifetime exist for larger film thickness, similarly to the experimental findings. On an absolute scale, the theoretical $T_{1 \text {,eff }}$ times appear to be longer than the corresponding experimental data, by typically $20 \%$ $25 \%$. At this point, one can stress that details of structural distortions of the substrate and the overlayer due to the Ag-Cu lattice mismatch as well as possible effects associated with the $9 \times 9$ superstructure of the silver film (for instance back folding of the Brillouin zone or existence of different adsorption sites) have not been taken into account; the present modeling involves perfectly flat surfaces and overlayers. In addition, the extraction of $T_{1 \text {,eff }}$ is very sensitive to the procedure used and to the choice of the actual laser-pulse shape. ${ }^{67,69}$ Still, despite these approximations, the present theoretical study catches the main features of the evolution of the $\mathrm{Cs}^{*}$ resonance as a function of the thickness of the thin Ag layer. This enables us to conclude a gradual change in the $\mathrm{Cs}^{*}$ dynamics from $\mathrm{Cu}$ to $\mathrm{Ag}$ substrates, the origin of which lies in the different efficiencies of the many-body relaxation of an excited electron in the two metals. 


\section{CONCLUDING SUMMARY}

We have reported on a joint experimental-theoretical study of the charge-transfer dynamics between a Cs adsorbate and an ultrathin $\mathrm{Ag}$ film deposited on a $\mathrm{Cu}(111)$ surface. An excited transient state is localized on the Cs adsorbate and decays by electron transfer into the substrate. The present study reveals the evolution of the electron transfer as the film thickness varies. The $\mathrm{Cs}^{*}$ resonance state corresponds to a $6 s-6 p$ hybrid and it exhibits a rather long lifetime in the 10-30 fs range at room temperature, similarly to what has been observed for bulk $\mathrm{Cu}(111)$ and bulk $\mathrm{Ag}(111)$ surfaces. The existence of a projected band gap beyond the Ag thin film is thus able to partly stabilize the $\mathrm{Cs}^{*}$ resonance in the same way as for a $\mathrm{Cu}(111)$ or $\mathrm{Ag}(111)$ substrate. The $\mathrm{Cs}^{*}$ resonance lifetime evolves as the film thickness is increased demonstrating the possibility to tune the adsorbate resonance lifetime. The evolution is monotonous from $\mathrm{Cu}$ to $\mathrm{Ag}$ and is attributed to the changes in the multielectron contribution to electron transfer. It is remarkable that beyond 2-3 ML, the
$\mathrm{Cs}^{*}$ resonance lifetime does not vary anymore. This is in variance with other surface properties such as, e.g., the surface state binding energy which varies over a broader range of film thickness [see Fig. 1(a); in that case, the broad range is attributed to the deep penetration of the surface state wave function into the solid]. One can thus say that the charge transfer is determined by the very first atomic planes of the solid. It also appears that the charge transfer between $\mathrm{Cs}^{*}$ and the substrate is not influenced significantly by changes in the surface electronic structure occurring at larger coverages, such as, e.g., the existence of quantum-well states.

\section{ACKNOWLEDGMENTS}

This work was supported by the Research Center OPTIMAS and the Deutsche Forschungsgemeinschaft through Grant No. DFG GRK 792 "Nichtlineare Optik und Ultrakurzzeitphysik." The work of V.M.S. is sponsored by the Ikerbasque foundation.
*Corresponding author: FAX: $\quad-49 \quad(0) 431 \quad 880 \quad 1685$; bauer@physik.uni-kiel.de.

${ }^{1}$ See for instance M. Haruta, Catal. Today 36, 153 (1997); M. Valden, X. Lai, and D. W. Goodman, Science 281, 1647 (1998).

${ }^{2}$ A. Ruban, B. Hammer, P. Stoltze, H. L. Skriver, and J. K. Nørskov, J. Mol. Catal. Chem. 115, 421 (1997).

${ }^{3}$ J. H. Larsen and I. Chorkendorff, Surf. Sci. 405, 62 (1998).

${ }^{4}$ J. H. Larsen and I. Chorkendorff, Catal. Lett. 52, 1 (1998).

${ }^{5}$ J. A. Rodriguez and D. W. Goodman, Science 257, 897 (1992).

${ }^{6}$ B. Hammer, Y. Morikawa, and J. K. Nørskov, Phys. Rev. Lett. 76, 2141 (1996).

${ }^{7}$ For an overview on QWS in metal films see for instance T.-C. Chiang, Surf. Sci. Rep. 39, 181 (2000).

${ }^{8}$ P. J. Rous, Phys. Rev. Lett. 83, 5086 (1999).

${ }^{9}$ A. G. Danese, F. G. Curti, and R. A. Bartynski, Phys. Rev. B 70, 165420 (2004).

${ }^{10}$ L. Aballe, A. Barinov, A. Locatelli, S. Heun, and M. Kiskinova, Phys. Rev. Lett. 93, 196103 (2004).

${ }^{11}$ N. Binggeli and M. Altarelli, Phys. Rev. Lett. 96, 036805 (2006).

${ }^{12}$ B. Sun, P. Zhang, S. Duan, X.-G. Zhao, and Q.-K. Xue, Phys. Rev. B 75, 245422 (2007).

${ }^{13}$ X. Ma, P. Jiang, Y. Qi, J. Jia, Y. Yang, W. Duan, W.-X. Li, X. Bao, S. B. Zhang, and Q.-K. Xue, Proc. Natl. Acad. Sci. U.S.A. 104, 9204 (2007)

${ }^{14}$ J. W. Gadzuk, L. J. Richter, S. A. Buntin, R. R. Cavanagh, and D. S. King, Surf. Sci. 235, 317 (1990).

${ }^{15}$ X.-L. Zhou, X.-Y. Zhu, and J. M. White, Surf. Sci. Rep. 13, 73 (1991).

${ }^{16}$ S. M. Harris, S. Holloway, and G. R. Darling, J. Chem. Phys. 102, 8235 (1995).

${ }^{17}$ D. C. Marinica, D. Teillet-Billy, J. P. Gauyacq, M. Michaud, and L. Sanche, Phys. Rev. B 64, 085408 (2001).

${ }^{18}$ J. W. Gadzuk, Annu. Rev. Phys. Chem. 39, 395 (1988).

${ }^{19}$ P. Haochang, T. C. M. Horn, and A. W. Kleyn, Phys. Rev. Lett. 57, 3035 (1986).
${ }^{20}$ A. G. Borisov, D. Teillet-Billy, and J. P. Gauyacq, Phys. Rev. Lett. 68, 2842 (1992).

${ }^{21}$ H. Winter, Comments At. Mol. Phys. 26, 287 (1991).

${ }^{22}$ S. Holloway and J. W. Gadzuk, J. Chem. Phys. 82, 5203 (1985).

${ }^{23}$ P. J. van der Hoek and E. J. Baerands, Surf. Sci. Lett. 221, L791 (1989).

${ }^{24}$ E. Yu. Usman, I. F. Urazgil'din, A. G. Borisov, and J. P. Gauyacq, Phys. Rev. B 64, 205405 (2001).

${ }^{25}$ M. Bauer, S. Pawlik, and M. Aeschlimann, Phys. Rev. B 55, 10040 (1997).

${ }^{26}$ S. Ogawa, H. Nagano, and H. Petek, Phys. Rev. Lett. 82, 1931 (1999).

${ }^{27}$ A. G. Borisov, A. K. Kazansky, and J. P. Gauyacq, Surf. Sci. 430, 165 (1999).

${ }^{28}$ A. G. Borisov, J. P. Gauyacq, A. K. Kazansky, E. V. Chulkov, V. M. Silkin, and P. M. Echenique, Phys. Rev. Lett. 86, 488 (2001); A. G. Borisov, J. P. Gauyacq, E. V. Chulkov, V. M. Silkin, and P. M. Echenique, Phys. Rev. B 65, 235434 (2002).

${ }^{29}$ For a review, see J. P. Gauyacq, A. G. Borisov, and M. Bauer, Prog. Surf. Sci. 82, 244 (2007).

${ }^{30}$ R. W. Gurney, Phys. Rev. 47, 479 (1935).

${ }^{31}$ J. Zhao, N. Pontius, A. Winkelmann, V. Sametoglu, A. Kubo, A. G. Borisov, D. Sanchez-Portal, V. M. Silkin, E. V. Chulkov, P. M. Echenique, and H. Petek, Phys. Rev. B 78, 085419 (2008).

${ }^{32}$ M. Bauer, S. Pawlik, R. Burgermeister, and M. Aeschlimann, Surf. Sci. 402, 62 (1998).

${ }^{33}$ W. E. McMahon, E. S. Hirschorn, and T.-C. Chiang, Surf. Sci. 279, L231 (1992).

${ }^{34}$ A. Bendounan, Y. Fagot-Revurat, B. Kierren, F. Bertran, V. Yu Yurov, and D. Malterre, Surf. Sci. 496, L43 (2002).

${ }^{35}$ W. Wallauer and Th. Fauster, Surf. Sci. 331-333, 731 (1995).

${ }^{36}$ M. Wessendorf, C. Wiemann, M. Bauer, M. Aeschlimann, M. A. Schneider, H. Brune, and K. Kern, Appl. Phys. A: Mater. Sci. Process. 78, 183 (2004).

${ }^{37}$ E. Bauer, Surf. Sci. 7, 351 (1967).

${ }^{38}$ A. Bendounan, H. Cercellier, Y. Fagot-Revurat, B. Kierren, V. 
Yu. Yurov, and D. Malterre, Phys. Rev. B 67, 165412 (2003).

${ }^{39}$ I. Meunier, G. Tréglia, J.-M. Gay, B. Aufray, and B. Legrand, Phys. Rev. B 59, 10910 (1999).

${ }^{40}$ M. A. Mueller, A. Samsavar, T. Miller, and T.-C. Chiang, Phys. Rev. B 40, 5845 (1989).

${ }^{41}$ K. Takahashi, A. Tanaka, H. Sasaki, W. Gondo, S. Suzuki, and S. Sato, Phys. Rev. B 60, 8748 (1999).

${ }^{42}$ S. Mathias, M. Wiesenmayer, M. Aeschlimann, and M. Bauer, Phys. Rev. Lett. 97, 236809 (2006).

${ }^{43}$ S. Mathias, M. Wessendorf, S. Passlack, M. Aeschlimann, and M. Bauer, Appl. Phys. A: Mater. Sci. Process. 82, 439 (2006).

${ }^{44}$ A. P. Shapiro, A. L. Wachs, and T.-C. Chiang, Solid State Commun. 58, 121 (1986).

${ }^{45}$ Physics and Chemistry of Alkali Adsorption, edited by H. P. Bonzel, A. M. Bradshaw, and G. Ertl (Elsevier, Amsterdam, 1989).

${ }^{46}$ H. Petek, H. Nagano, M. J. Weida, and S. Ogawa, J. Phys. Chem. B 105, 6767 (2001).

${ }^{47}$ M. Bauer, S. Pawlik, and M. Aeschlimann, Phys. Rev. B 60, 5016 (1999).

${ }^{48}$ R. Paniago, R. Matzdorf, G. Meister, and A. Goldmann, Surf. Sci. 336, 113 (1995).

${ }^{49}$ S. D. Kevan, Phys. Rev. Lett. 50, 526 (1983).

${ }^{50}$ S. D. Kevan, Phys. Rev. B 33, 4364 (1986).

${ }^{51}$ X. Y. Wang, R. Paiella, and R. M. Osgood, Jr., Phys. Rev. B 51, 17035 (1995).

${ }^{52}$ M. Weinelt, Ch. Reuß, M. Kutschera, U. Thomann, I. L. Shumay, Th. Fauster, U. Höfer, F. Theilmann, and A. Goldmann, Appl. Phys. B: Lasers Opt. 68, 377 (1999).

${ }^{53}$ A. G. Borisov, J. P. Gauyacq, and A. K. Kazansky, Surf. Sci. 505, 260 (2002).

${ }^{54}$ N. Pontius, V. Sametoglu, and H. Petek, Phys. Rev. B 72, 115105 (2005).

${ }^{55}$ P. Tegeder, S. Hagen, F. Leyssner, M. V. Peters, S. Hecht, T. Klamroth, P. Saalfrank, and M. Wolf, Appl. Phys. A: Mater. Sci. Process. 88, 465 (2007).
${ }^{56}$ E. V. Chulkov, A. G. Borisov, J. P. Gauyacq, D. Sanchez-Portal, V. M. Silkin, V. P. Zhukov, and P. M. Echenique, Chem. Rev. 106, 4160 (2006).

${ }^{57}$ J. N. Bardsley, Case Stud. At. Phys. 4, 299 (1974).

${ }^{58}$ L. Kleinman and D. M. Bylander, Phys. Rev. Lett. 48, 1425 (1982).

${ }^{59}$ M. Caragiu, G. S. Leathermann, R. D. Diehl, P. Kaukasoina, and M. Lindroos Surf, Science 441, 84 (1999).

${ }^{60}$ E. V. Chulkov, V. M. Silkin, and P. M. Echenique, Surf. Sci. 437, 330 (1999).

${ }^{61}$ J. Kliewer, R. Berndt, E. V. Chulkov, V. M. Silkin, P. M. Echenique, and S. Crampin, Science 288, 1399 (2000).

${ }^{62}$ E. V. Chulkov, J. Kliewer, R. Berndt, V. M. Silkin, B. Hellsing, S. Crampin, and P. M. Echenique, Phys. Rev. B 68, 195422 (2003).

${ }^{63}$ A. G. Borisov, A. K. Kazansky, and J. P. Gauyacq, Phys. Rev. B 59, 10935 (1999).

${ }^{64}$ L. Hedin and S. Lundqvist, in Solid State Physics, edited by H. Ehrenreich, F. Seitz, and D. Turnbull (Academic Press, New York, 1969), Vol. 23, p. 1.

${ }^{65}$ P. M. Echenique, R. Berndt, E. V. Chulkov, Th. Fauster, A. Goldman, and U. Höfer, Surf. Sci. Rep. 52, 219 (2004).

${ }^{66}$ H. Petek, M. J. Weida, H. Nagano, and S. Ogawa, Science 288 , 1402 (2000).

${ }^{67}$ M. Bauer, M. Wessendorf, D. Hoffmann, C. Wiemann, A. Mönnich, and M. Aeschlimann, Appl. Phys. A: Mater. Sci. Process. 80, 987 (2005).

${ }^{68}$ A. G. Borisov, A. K. Kazansky, and J. P. Gauyacq, Phys. Rev. B 64, 201105 (2001).

${ }^{69}$ J. P. Gauyacq and A. K. Kazansky, Phys. Rev. B 72, 045418 (2005).

${ }^{70}$ H. Petek and S. Ogawa, Prog. Surf. Sci. 56, 239 (1997).

${ }^{71}$ Note that in Fig. 9 of Ref. 69 the experimental results for the Gaussian and the hyperbolic secant pulse shapes have been exchanged by mistake. 